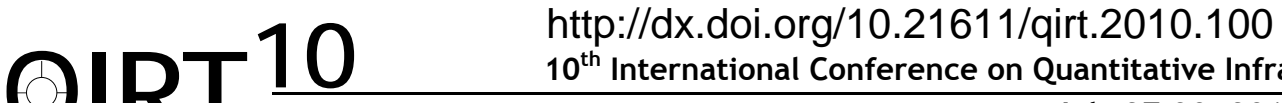 \\ $10^{\text {th }}$ International Conference on Quantitative InfraRed Thermography \\ July 27-30, 2010, Québec (Canada)
}

\section{IR Non Destructive Testing Experiment Design}

\author{
By Dan Necsulescu*and Sharareh Bayat *and Mohammad Eghtesad** \\ *Mech. Eng. Dept., University of Ottawa, Ottawa (Ontario) Canada K1N 6N5, necsu@uottawa.ca, sbaya016@uottawa.ca \\ ${ }^{*}$ Mech. Eng. Dept., Shiraz University, Shiraz, Iran, eghtesad@shirazu.ac.ir
}

\begin{abstract}
Non destructive testing requires the predetermination of the parameters of the experiment. In this paper the focus is on the design of the experiments for infrared testing based on analytical models. Resulting design parameters are obtained using conservative approximate equations. The results are particularly relevant for non destructive testing in thin plates of various materials

\section{Introduction}

The goal of this paper is to develop equations that permit the design of experiments for non-destructive infrared testing. These equations permit the predetermination of the frequency for the input thermal signal given the material defect size and the first time when steady state values can be recorded. Infrared testing is used for obtaining thermal images influenced by internal defects. In the proposed approach the model used for the experimental design of non-destructive infrared active testing of materials is reduced from three-dimensional to one-dimensional. The results, recorded before reflected thermal waves from more remote boundaries arrive back to the point under investigation, can be used for defect detection. Simulation results illustrate the usefulness of the pre-determination of the characteristics of the input thermal signal for the investigation of possible defects in materials.
\end{abstract}

\section{Direct problem formulation for heat flow}

Direct problem is formulated for one-dimensional heat conduction problem by the non-homogenous equation [1]

$u(x, y, z, t)=\alpha \cdot \nabla^{2} u(x, y, z, t-\Delta t)+F(x, y, z, t-\Delta \mathrm{t})$

Where:

$\mathrm{u}(\mathrm{x}, \mathrm{t})$ is the temperature of a solid body in the point $\mathrm{x}$ at time $\mathrm{t}$.

$\alpha$ is the diffusivity coefficient given by

$\alpha=k /(\sigma . \tau)$

where $k$ is the thermal conductivity $[\mathrm{W} /(\mathrm{m} \cdot \mathrm{K})]$,

$\sigma$ is the specific heat of the solid body conducting the heat $[\mathrm{J} /(\mathrm{kg} \cdot \mathrm{K})]$

$\tau$ is the volume density $\left[\mathrm{kg} / \mathrm{m}^{3}\right]$

$F(x, y, z, t)$ is heat density (or heat flux) from a heat source.

Direct problem refers to the effects of heat density or distributed heat flux $F(x, t)$ sources on the distributed body temperature $u(x, t)$.

Boundary conditions are specific to each particular case. Initial conditions take the form

$u(x, t)=\varphi(x)$

The method of separation of variables leads to the solution $u(x, t)=X(x) . T(t)$. The solution for the direct problem is not in closed form and this results in major difficulties in real-time solution of the inverse problem for determining $F(x, t)$ for achieving a desired $\mathrm{u}(\mathrm{x}, \mathrm{t})$. Moreover, often the external heat source or the external temperature input is on the boundary and is included in the boundary conditions.

This paper investigates the limits of validity of a 1D heat transfer model for the design of the experiments in non-destructive infrared testing for 2D and 3D materials.

The boundary temperature at $x=0$ is assumed to be cosinusoidal $u(0, t)=U \cos (i \omega t-\varepsilon)$

where $U$ is the amplitude of the temperature oscillation. The value of the parameter $-\varepsilon$ is the initial phase of $u(0, t)$ For a semi-infinite solid $(x \geq 0)$ and initial temperature $u(0, t)=0$, the solution of Eq. 1is composed of a steady state $u_{s s}$ and a transient part $\mathrm{u}_{\mathrm{tr}}[1]$

$u(x, t)=u_{s s}(x, t)+u_{t r}(x, t)$

where 
$u_{t r}(x, t)=-\frac{2 U}{\sqrt{\pi}} \int_{0}^{\frac{x}{2 \sqrt{\alpha t}}} \cos \left(\omega\left(t-\frac{x^{2}}{4 \alpha \mu^{2}}\right)-\varepsilon\right) e^{-\mu^{2}} d \mu$

$u_{s s}(x, t)=\frac{2 U}{\sqrt{\pi}} \int_{0}^{\infty} \cos \left(\omega\left(t-x \sqrt{\frac{\omega}{2 \alpha}}\right)-\varepsilon\right) e^{-\mu^{2}} d \mu$

The definite integral in (6) yields to [1]

$u_{S S}(x, t)=U e^{-x \sqrt{\omega / 2 \alpha}} \cos \left(\omega t-x \sqrt{\frac{\omega}{2 \alpha}}-\varepsilon\right)$

In which the coefficient of $\mathrm{x}$ is the wave number $\mathrm{K}$ given by

$K=\sqrt{\omega / 2 \alpha}$

\subsection{Determination of the Duration $t_{2 \%}(x)$ of the Transient Part $u_{t r}(x, t)$}

The transient part, $\mathrm{u}_{\mathrm{tr}}(\mathrm{x}, \mathrm{t})$, vanishes as $\mathrm{t} \rightarrow \infty$, when that happens the upper limit results in $\lim _{\mathrm{t} \rightarrow \infty} x / \sqrt{\alpha t} \rightarrow 0$ and the integral tends towards zero. The transient regime can be considered practically finished when its contribution is less than $2 \%$ of the steady state value of $u(x, t)$. For this purpose, an approximate form is useful. First, given that

$-1 \leq \cos \left\{\omega\left(t-\frac{x^{2}}{4 \alpha \mu^{2}}-\varepsilon\right\} \leq 1\right.$

Eq. (5) takes values within the limits

$-\frac{2 U}{\sqrt{\pi}} \int_{0}^{\frac{x}{2 \sqrt{a \pi}}} e^{-\mu^{2}} d \mu \leq u_{t r}(x, t) \leq \frac{2 U}{\sqrt{\pi}} \int_{0}^{\frac{x}{2 \sqrt{a \pi}}} e^{-\mu^{2}} d \mu$

The integral is a bell shaped Gauss function, symmetric with regard to $\mu$. For any positive values of $\mu, \mathrm{e}^{-\mu^{2}}<1$ therefore, Eq. 9 turns into

$-\frac{U_{x}}{\sqrt{\pi \alpha t}} \leq u_{t r}(x, t) \leq \frac{U_{x}}{\sqrt{\pi \alpha t}}$

or

$\left|u_{t r}(x, t)\right| \leq \frac{U_{x}}{\sqrt{\pi \alpha t}}$

Which tends toward 0 as $t \rightarrow \infty$.

The steady state harmonic part, $u_{s s}(x, t)$, is the value of $u(x, t)$ after the end of the transient regime. In fact, for 1D heat transfer, there is a closed form solution given by $(7)$ which gives the amplitude of $u_{s s}(x, t)$

$\left|u_{s S}(x)\right|=U e^{-x \sqrt{w / 2 \alpha}}$

The $2 \%$ settling time is, in this case, defined by

$\frac{U_{x}}{\sqrt{\pi \alpha t_{2 \%}}}=0.02 U e^{-x \sqrt{\omega / 2 \alpha}}$

This gives the equation for obtaining a conservative approximation of the $2 \%$ settling time

$t_{2 \%}(x)=\frac{1}{\pi k}\left(\frac{x}{0.02 e^{-x \sqrt{\omega / 2 \alpha}}}\right)^{2}$

For $\mathrm{x}=0.1[\mathrm{~mm}]$ and $\omega=0.1[\mathrm{rad} / \mathrm{s}], \mathrm{t}_{2} \%$ results in $0.093[\mathrm{~s}]$ for aluminum and 69.297 [s] for rubber.

In Fig. 1 are shown the results for $t_{2} \%$ at $x=0.3[\mathrm{~mm}]$ for (a) aluminum and (b) rubber as functions of $\omega$. The outcomes indicate that selecting lower values for $\omega$ result in shorter settling times. 


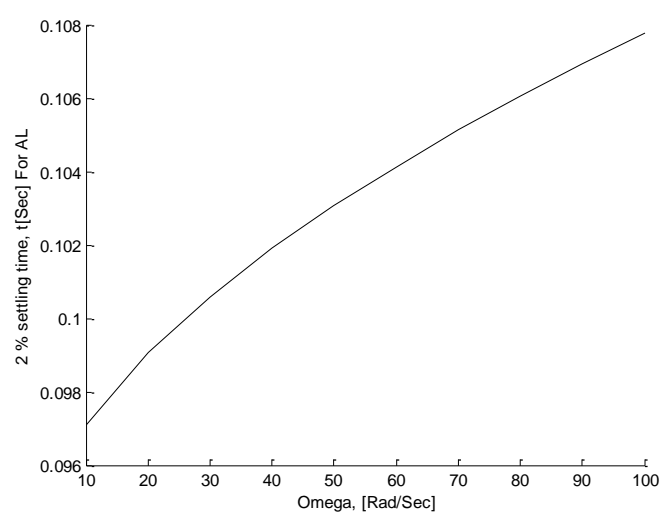

(a)

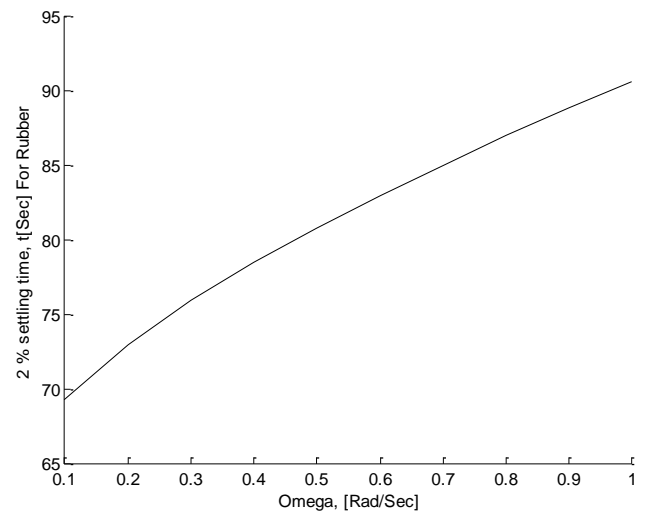

(b)

Fig. 1. Settling time $t_{2} \%$ at $x=0.3[\mathrm{~mm}]$ for (a) Aluminum and (b) Rubber vs. $\omega$ (Input Frequency)

\subsection{Determination of the Frequency $\omega$ of the Excitation Signal}

The amplitude of $u_{s s}(x, t)$ from Eq. 12 shows an exponential decrease with $x$ and $\sqrt{ } \omega$. This indicates that, in order to compensate for the exponential decay of $\left|u_{s s}(x)\right|$ with the increase of distance $x$, a reduction of the radial frequency $\omega$ of the input $u(0, t 0=U \cos (\omega t-\varepsilon)$ can be effective.

Eq. 12 gives

$\omega=\frac{2 k}{x^{2}}\left[\ln \left\{\left|u_{s s}(x)\right|\right\}\right]^{2}$

Fig. 2 illustrates the input signal frequency $\omega$, as a function of variations in the ratio of $\left|u_{s s}(x)\right|$ and the input signal amplitude $\mathrm{U},\left(\left|U_{s S}(x)\right| / U\right)$, at $\mathrm{x}=0.3[\mathrm{~mm}]$, for (a) aluminum and (b) rubber. In accordance to simulation results, selecting higher values of $\left|U_{s s}(x)\right| / U$ will result in lower values of $\omega$. Namely smaller amounts of input frequency yields in steady state outputs with the amplitudes which are easier to measure accurately.

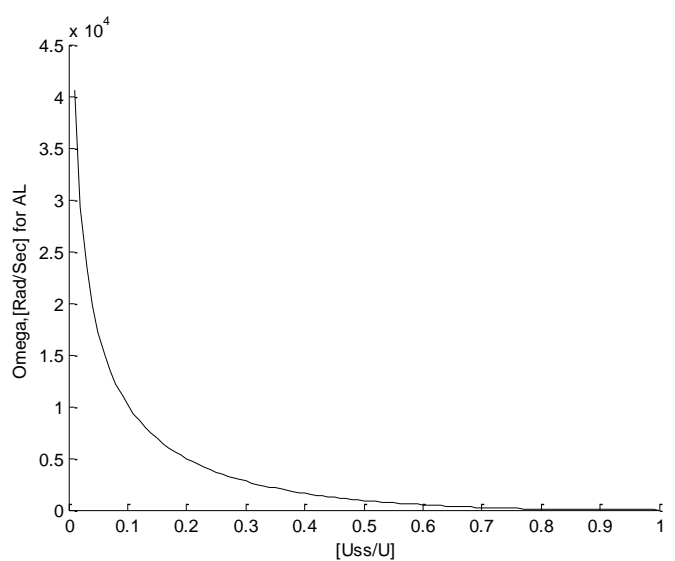

(a)

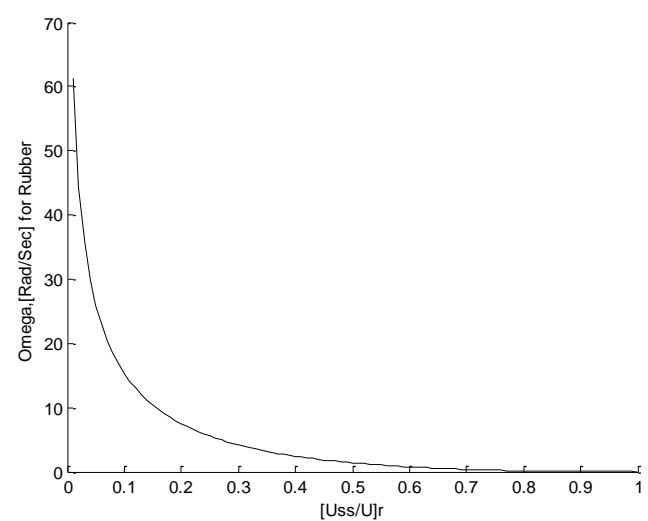

(b)

Fig. 2. Input frequency $\omega$ at $x=0.3[\mathrm{~mm}]$ for (a) Aluminum and (b) Rubber function vs. $/ u_{s s}(x) / / U$

\subsection{Determination of the minimum time $\Theta$ required for $u_{s s}(x, t)$ measurement}

Given the period $\mathrm{T}=2 \pi / \omega$ of the excitation signal, the determination of its amplitude $\left|\mathrm{u}_{\mathrm{ss}}(\mathrm{x})\right|$ given by Eq. 12 and of its phase difference, $\mathrm{Kx}$, with regard to $\mathrm{u}(0, \mathrm{t})$, the duration $\Theta$ of the measurement has to cover at least two extreme values and two zero crossings, i.e. at least one period $\mathrm{T}$ of the cosinusoidal signal, to reduce the effect of measurement noise, i.e. 
$\Theta>\frac{\lambda}{v}=\frac{\lambda}{\sqrt{2 \alpha \omega}}$

Fig. 3 shows the variations of the minimum time required for $u_{s s}(x, t)$ measurement for (a) aluminum and (b) rubber, function of the input signal frequency $\omega$.

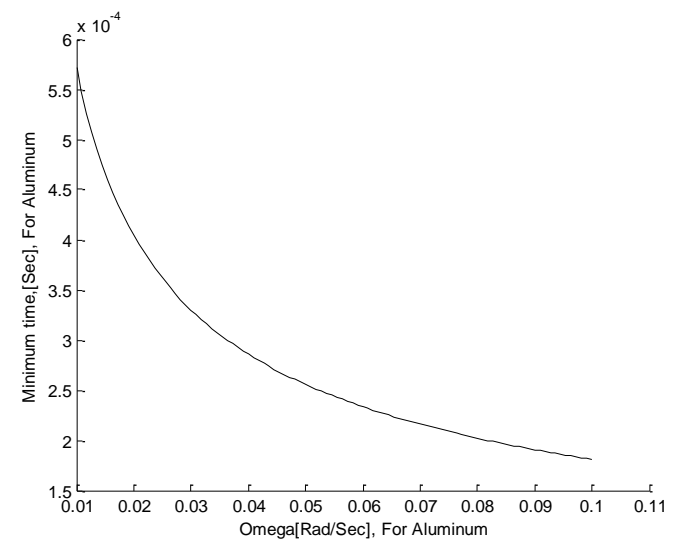

(a)

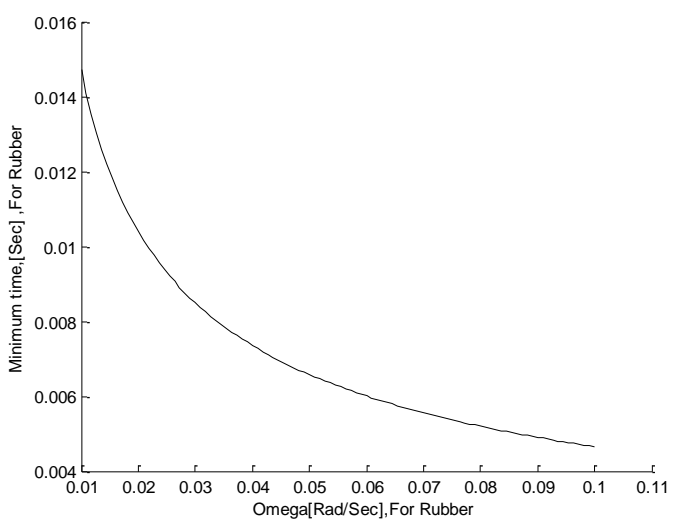

(b)

Fig. 3. Minimum time required for $u_{s s}(x, t)$ measurement for (a) Aluminum and (b) Rubber vs. $\omega$ (Input frequency)

\subsection{Determination of the Time $\Delta t$ for First Reflection}

Referring to equation (7) and considering the general form of electromagnetic wave propagation, the relationship between the wave number and the corresponding velocity is described as:

$k \equiv \frac{2 \pi}{\lambda}=\frac{2 \pi \nu}{V}=\frac{\omega}{v_{p}}$

Where $v$ is the frequency of the thermal wave, $V$ is the velocity of the wave and $\omega$ is the angular frequency of the wave.

It can be seen that the velocity $V$ of the thermal wave propagation is given by the ratio of the coefficients of $t$ and $x$ from Eq. (7) as $\omega / \sqrt{ }(\omega / 2 k)$, i.e.

$V=\sqrt{(2 k \omega)}$

For an excitation signal with radial frequency $\omega$ and period $T=2 \pi / \omega$

$V=\sqrt{(4 \pi k / T)}$

Assuming that the plate has the dimensions $Y$ along $y$ and $Z$ along $z$, and that the closest boundary of the plate along $y$ and $z$ is $\mathrm{m}=\min \{\mathrm{Y}, \mathrm{Z}\}$, therefore the earliest time for the first reflection on the boundary is given by

$\Delta t=\frac{m}{V}=m / \sqrt{(2 k \omega)}$

For $t>\Delta t$, the signal $\mathrm{u}(\mathrm{x}, \mathrm{t})$ is composed not only of the direct thermal wave, but also of the reflected thermal waves and, consequently, becomes more complex and difficult to analyze for non-destructive testing.

Fig. 4 shows the variations of the earliest time for the first reflection on the boundary, $\Delta t$, for $m=1$, as a function of the input signal frequency $\omega$ for both aluminum and rubber,. 


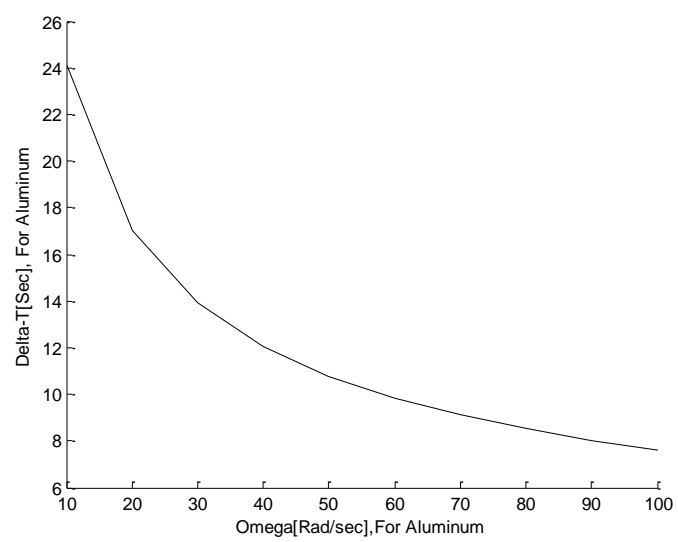

(a)

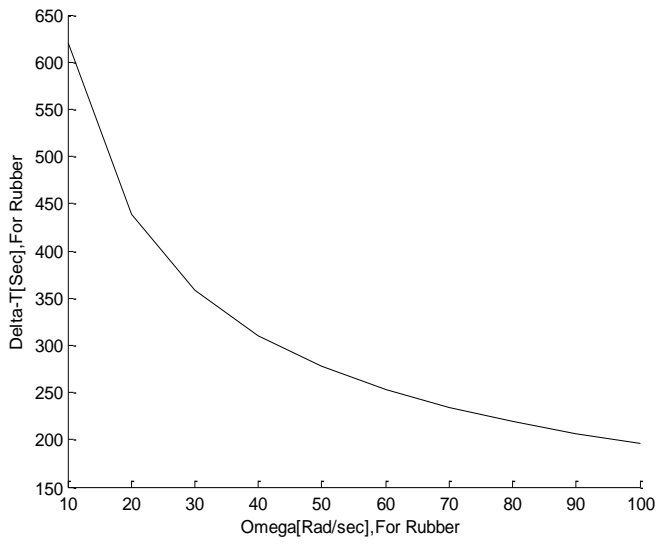

(b)

Fig. 4. The relationship between the first reflection time $\Delta t$ and input frequency $\omega$ for a) Aluminum, and (b) Rubber, the shortest distance to the boundary $m$ is assumed unity)

\section{Simulation Results for $1 \mathrm{D}$ Approach of Non-destructive Infrared Testing}

In this paper the possibility of using 1D heat transfer approximation for the design of the experiments in nondestructive infrared testing of 2D and 3D materials is investigated. This approximation is based on assuming that $2 \%$ settling time of $u_{t r}(x, t)$ is short enough that the test can be focused on steady state harmonic, $u_{s s}(x, t)$, part of $u(x, t)$ to characterize defects in materials. In this simulation, the defect investigated is a variation of the width $L$ of a plate/beam about a nominal value, as a result of material processing variations. These simulations were carried out to determine the efficient radial frequency $\omega$ of the input cosinusoidal signal $u(0, t)$, given the material type characterized by thermal diffusivity coefficient $\alpha$ and the nominal width $\mathrm{L}$.

For the case of a sinusoidal heat source with the amplitude of 1 at $x=0[m], u(0, t)=\cos (\omega t)$, the simulation results of the steady state harmonic temperature $u_{s s}(x, t)$ are shown in Fig. 5 and 6 , for aluminum and rubber. Thermal diffusivity coefficients, $\alpha$, are $1.3 \times 10^{-7}\left[\mathrm{~m}^{2} / \mathrm{s}\right]$ for rubber and $8.6 \times 10^{-5}\left[\mathrm{~m}^{2} / \mathrm{s}\right]$ for aluminum. In Fig. 5 , at $\mathrm{x}=\mathrm{L}=0.01[\mathrm{~m}]$, for aluminum the amplitude of the signal is much smaller than at $x=0[\mathrm{~m}]$, but still can be measured. For rubber, because of the smaller diffusivity, the amplitude of the signal at $x=0.01[\mathrm{~m}]$ is extremely small, almost close to zero, and is not measurable any more. The results in Fig. 6 show that, for rubber, even by decreasing $\omega$ to 0.05 [rad/s], still no signal reaches the other end, and only for the frequency $\omega$ equals to $0.01[\mathrm{rad} / \mathrm{s}]$, a very small amplitude results at $x=0.01[\mathrm{~m}]$.

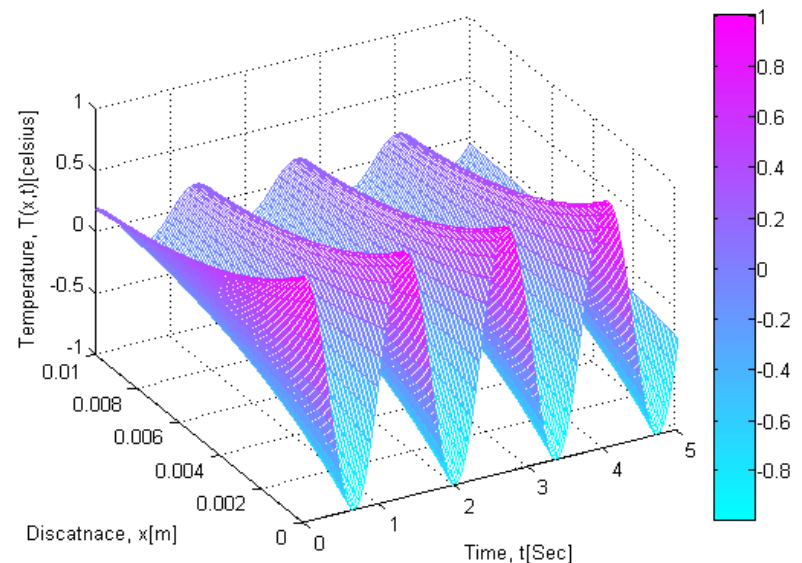

Aluminum

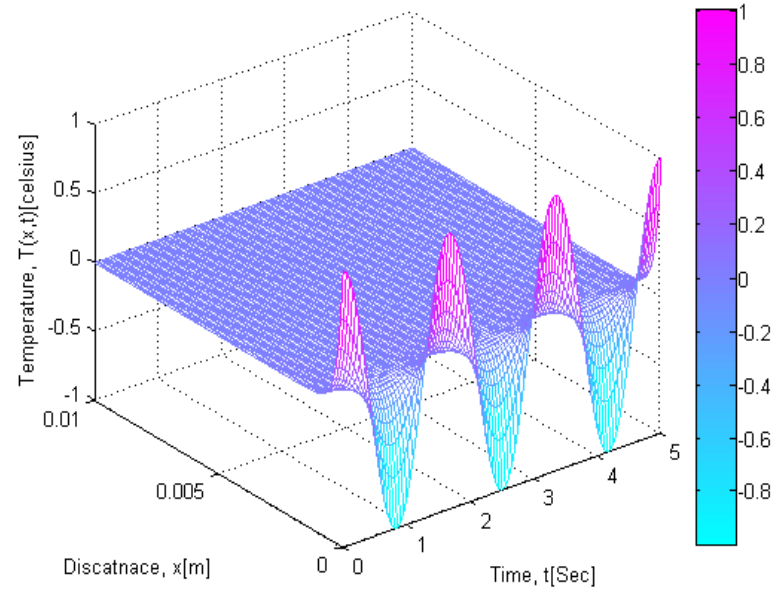

Rubber

Fig. 5. Steady state harmonic temperature $u_{s s}(x, t)$ for $L=0.01[\mathrm{~m}]$ and $\omega=5[\mathrm{rad} / \mathrm{s}]$ 


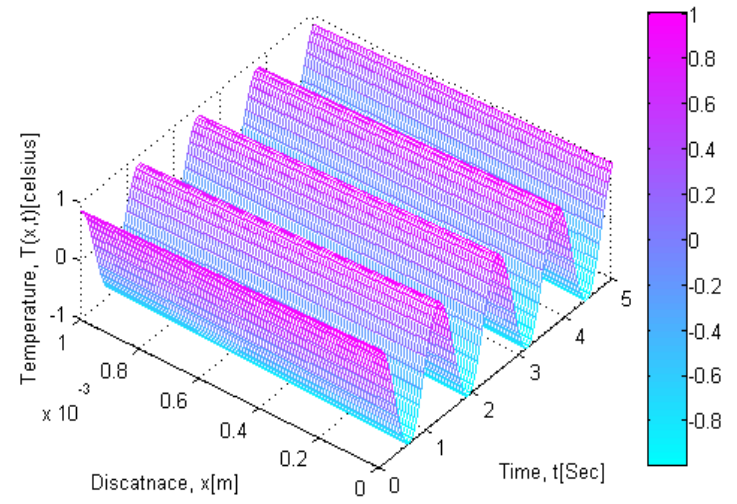

Aluminum

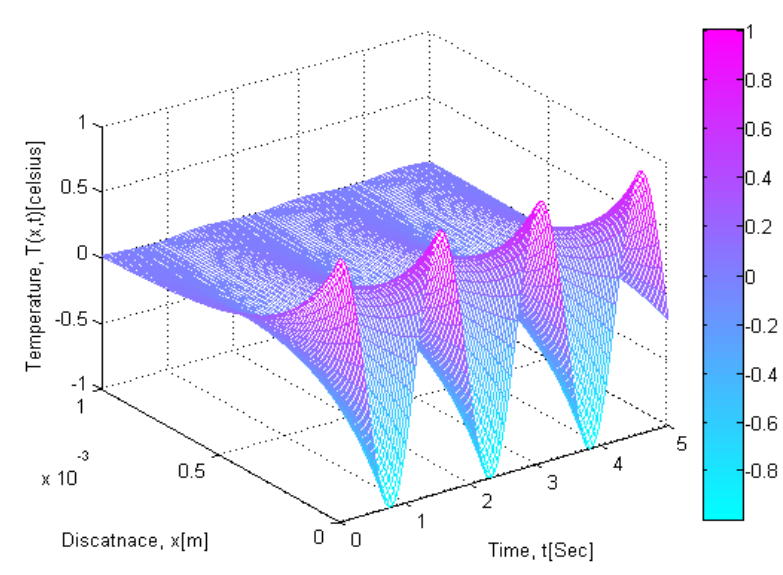

Rubber

Fig. 6 Steady state harmonic temperature $u_{s s}(x, t)$ for $L=0.001[\mathrm{~m}]$ and $\omega=5[\mathrm{rad} / \mathrm{s}]$

\section{Conclusions}

Prior experimental design of the experiments for infrared non destructive testing requires for the choice of test parameters. The paper presented the methodology tor obtain the radial frequency of the external temperature variation for a desired ratiol $u_{s s}(x, t) / U$, for obtaining the time when the measurement of $u_{s s}(x, t)$ can be effective, i.e. after the end of the transient regime, and for the calculation of an conservative upper limit of the $2 \%$ settling time. Simulation results illustrate the usefulness of these theoretical results.

\section{REFERENCES}

[1] Carlslaw H. and Jaeger J., Conduction of Heat in Solids, Oxford, 1959.

[2] Betounes D., Partial Differential Equations for Computational Science with Maple and Vector Analysis, Springer, 1998.

[3] Necsulescu D., Advanced Mechatronics, World Scientific, 2009.

[4] Necsulescu D., Bayat S.and Dinca A., Investigation of Non Destructive Infrared Testing Issues using Inverse Problem Formulation, XIV Scientific Conference, C. Brâncuşi University of Tg-Jiu, Nov., 2009.

[5] Shih T-H., Numerical Heat transfer, Hemishere Publishing Corporation, 1984.

[6] Grigull U., Sandner H., Heat Conduction, Springer, 1984.

[7] Murio D., The Mollification Method and the Numerical Solution of III-Posed Problems, Wiley, 1993.

[8] V. Ghali et al., Three-Dimensional Pulse Compression for IR Teting, IEEE Sensors Journal, Vol. 9, No. 7, July 2009, pp. 832-834.

[9]Doumanidis C., Thermomechatronics, in C. Leondes (Ed), Mechatronic Systems Techniques and Applications, Vol. 1, Industrial Manufacturing, Gordon and Breach Science Publishers, 2000, pp. 255-313.

[10] Vavilov V. A., Advances in Signal Inversion with Applications to Infrared Thermography, in Advances in Signal Processing for Nondestructive Evaluation of Materials, X. Maldague (ed), Kluwer, 1994, pp. 161-184.

[11] Maldague X. (Editor), Advances in Signal Processing for Nondestructive Evaluation of Meterials, Kluwer, 1994.??

[12] Maldague X., Theory and Practice of IR Technology for Nondestructive Testing, Wiley, 2001.

[13] Necsulescu D. and Sasiadek J., Indirect Mesurement using Inverse Problems Solutions, Proc. ICCC'08 Conference, Sinaia, 25-28 May, 2008, pp. 438-441.

[14] Beck J. et al., Inverse Heat Conduction. III posed problems, Wiley, 1985. 